\title{
REFLEXIONES SOBRE EL ACCESO A LOS SERVICIOS DE SALUD \\ DE LAS PERSONAS CON DISCAPACIDAD \\ INTELECTUAL O DEL DESARROLLO \\ EN EL MARCO DE LA CRISIS SANITARIA CREADA POR LA PANDEMIA DE LA COVID-19
}

\section{Reflections on Access to Health Services for People with Intellectual or Developmental Disabilities in the Context of the Health Crisis created by the COVID-19 Pandemic}

Javier MuÑoz BRAvo

Instituto de Salud Carlos III. Madrid

jmunoz@ciberes.org

Inés de Araoz Sánchez-Dopico

Plena inclusión España

Recepción: 30 de octubre de 2020

Aceptación: 1 de febrero de 2021

Resumen: La pandemia de COVID-19 tuvo en su primera oleada entre marzo y mayo de 2020 un impacto enorme sobre el sistema sanitario español. La saturación del mismo y la escasez de recursos materiales y personales obligaron a tomar decisiones que implicaban la priorización de la atención de unos pacientes sobre otros. La toma de estas decisiones se hizo en la mayoría de los casos sin tomar en consideración las características, las necesidades y los derechos de las personas con discapacidad intelectual y del desarrollo cuando no basadas en sesgos negativos sobre el colectivo. Se analiza la situación creada durante el primer pico pandémico y se extraen conclusiones relevantes para la preservación del derecho a la salud.

Palabras Clave: derecho a la salud; COVID-19; discapacidad intelectual; SARSCoV-2; bioética. 
Aвstract: The COVID-19 pandemic had in its first wave between March and May 2020 , and had a huge impact on the Spanish health system. Its saturation and the scarcity of material and personal resources forced decisions which implied prioritizing the care of some patients over others. These decisions were made in most cases without taking into account the characteristics, needs and rights of people with intellectual and developmental disabilities, when not based on negative biases on the group. The situation created during the first pandemic peak is analyzed and relevant conclusions are drawn for the preservation of the right to healthcare access. ethics.

KEYwORDs: right to health; COVID-19; intellectual disability; SARS-CoV-2; bio-

\section{Introducción}

$\mathrm{P}$ ARA COMPRENDER MEJOR LA SITUACIÓN de la atención sanitaria de las personas con discapacidad intelectual o del desarrollo (en adelante personas con DID) por la pandemia global por SARS-CoV-2, consideramos que deben aportarse algunos datos que pongan en evidencia la excepcionalidad y la gravedad de la situación que se generó en España entre los meses de marzo y mayo de 2020; situación similar a la generada en otros lugares del mundo antes y después de esas fechas.

El 31 de diciembre de 2019, China reportó a la Organización Mundial de la Salud 27 casos de personas enfermas que padecían un tipo de neumonía de causa desconocida, 7 de ellos en condición crítica, en Wuhan, provincia de Hubei. Solo un mes después, el 31 de enero de 2020, la OMS declaró el brote de SARS-CoV-2 como emergencia de Salud Pública de importancia internacional y el 11 de marzo fue declarada pandemia global. La enfermedad causada por este nuevo virus se ha denominado por consenso internacional COVID-19.

En su primera ola, entre marzo y mayo de 2020, la COVID-19 puso en serio riesgo de colapso al sistema sanitario español. Y, según la Red Nacional de Vigilancia Epidemiológica del Instituto de Salud Carlos III en su informe 54 de 25 de noviembre de 2020 RENAVE (2020), los casos notificados a la RENAVE con diagnóstico posterior al 10 de mayo de 2020 ascendían a 1.371.376, siendo necesaria la hospitalización de 79.671 personas.

El rápido incremento de los contagios y la saturación de los servicios de salud obligaron a las autoridades españolas a implementar medidas urgentes en el ámbito de la salud pública, fundamentalmente el confinamiento de la población y la restricción de su movilidad que, de manera general, permitieron reducir el número de casos nuevos hasta niveles que llevaron a poder disponer de la capacidad hospitalaria necesaria y el suficiente personal sanitario debidamente cualificado y protegido para atender la demanda de atención generada por le enfermedad.

Los datos de la Tabla 1 ayudan a entender cómo entre los meses de abril y mayo de 2020 el sistema sanitario español estuvo al borde del desplome, especialmente en las zonas más afectadas por la pandemia (Comunidad Autónoma de Madrid, Cataluña y algunas capitales de Castilla y León). Esta saturación del sistema sanitario cristalizó 
en la imposibilidad de prestar la atención adecuada a las necesidades de cada paciente y en la obligación de priorizar la asistencia de unos pacientes sobre otros. También se puede observar cómo la presión asistencial comenzó a incrementarse nuevamente de forma exponencial a partir de septiembre.

\begin{tabular}{|c|c|c|}
\hline $\begin{array}{r}\text { TABLA 1. Evolución del número de personas que han requerido hospitalización } \\
\text { por la COVID-19 en España entre febrero y }\end{array}$ \\
\hline & Hospitalizadas & UCI \\
\hline $28 / 02 / 2020$ & 17 & 2 \\
\hline $26 / 03 / 2020$ & 9.759 & 737 \\
\hline $4 / 05 / 2020$ & 78.145 & 4.160 \\
\hline $3 / 09 / 2020$ & 12.109 & 878 \\
\hline $7 / 10 / 2020$ & 30.709 & 2.390 \\
\hline $28 / 10 / 2020$ & 48.211 & 3.804 \\
\hline $26 / 11 / 2020$ & 79.671 & 6.388 \\
\hline
\end{tabular}

Las consecuencias de la priorización de los pacientes debida a la sobrecarga de los servicios de salud sobre el derecho a la salud de las personas con DID son las que centran el contenido de este artículo.

2. La incidencia de la COVID-19 en las personas con discapacidad intelectual y del desarrollo

De acuerdo con los datos ofrecidos por el Ministerio de Sanidad (2020) en relación a la COVID-19, se considera que existen personas o grupos de personas que pueden ser más vulnerables a la COVID-19 o con mayor riesgo de desarrollar enfermedad grave. Este mayor riesgo se relaciona con tener más de 60 años, enfermedades cardiovasculares e hipertensión arterial, diabetes, enfermedades pulmonares crónicas, cáncer, inmunodepresión o estar embarazada.

Coincidiendo con estos criterios, según los datos notificados a la RENAVE, informe 33, hasta el 10 de mayo el $86 \%$ de los pacientes que falleció tenían más de 70 años, el $95 \%$ de los mismos presentaban algún tipo de enfermedad de base previa y el $60 \%$ padecían una enfermedad cardiovascular. La prevalencia de enfermedad de base es del $51 \%$ en los casos no hospitalizados, ascendiendo al $79 \%$ en los casos hospitalizados, al $81 \%$ en los ingresados en UCI y el $95 \%$ en los fallecidos, siendo la enfermedad cardiovascular la que presenta mayor prevalencia entre los pacientes de COVID-19 hospitalizados (41\%), en UCI (43\%) y fallecidos (60\%). A partir del 10 de mayo, a fecha de 25 de noviembre de 2020, el informe 54 refleja que el 46,7 \% de las 
personas hospitalizadas tenían más de 70 años, siendo la prevalencia del fallecimiento del $85,4 \%{ }^{1}$.

La obesidad también es un factor que puede jugar un papel importante en la infección por COVID-19. Según Fernández Bretón (2020), en un análisis retrospectivo de 112 pacientes ingresados en Wuhan por COVID-19, el IMC de los pacientes críticos fue significativamente superior al del grupo general (25,5 frente a 22,0$)$. La prevalencia de obesidad (IMC > 25) entre los que fallecieron fue del 84,8\% frente al 18,9\% en pacientes que superaron la enfermedad.

Según el Ministerio de Sanidad (2020) también se consideran más vulnerables las personas que viven o trabajan en instituciones cerradas, con especial atención a las personas mayores que viven en residencias, debido a que este tipo de instituciones en las que se comparten espacios comunes y en las que conviven muchas personas hacen más compleja la prevención del contagio.

De acuerdo a la definición de la Asociación Americana de Discapacidades Intelectuales y del Desarrollo (2011), la discapacidad intelectual se caracteriza por limitaciones significativas tanto en el funcionamiento intelectual como en la conducta adaptativa tal y como se manifiesta en habilidades adaptativas conceptuales, sociales y prácticas.

Por tanto, de acuerdo con lo anterior las personas con DID no son, por causa de la discapacidad intelectual, personas con mayor riesgo de desarrollar enfermedad grave por COVID-19 salvo que concurra alguna de las circunstancias anteriores, es decir, presenten algún factor de riesgo o vivan en instituciones cerradas.

A pesar de ello, es preciso tener en cuenta que sí existen evidencias de una mayor incidencia de algunos de estos factores de riesgo en personas con DID. Según Folch et al. (2019), un 8,10 \% de la población con discapacidad intelectual presenta cardiopatías (incluyendo infarto, angina de pecho y enfermedad coronaria) frente a un 5,21\% de la población general; un 8,7 \% presentaría enfermedad respiratoria crónica frente a un 3,32 \% de la población general, y un 7,5\% diabetes frente a un 6,84 \% en el resto de ciudadanos.

Los problemas de sobrepeso y obesidad han sido reportados en gran cantidad de estudios sobre DI. Según Muñoz Bravo et al. (2014), la obesidad es más alta en las personas con DI en comparación con la población general, en concreto en la muestra del estudio realizado sobre indicadores del estado de salud de personas con discapacidad intelectual se halló que el 32,8 \% y el 29,9 \% de las personas tenían sobrepeso y obesidad respectivamente.

1 Los informes de la RENAVE sobre la situación de COVID-19 se suspendieron a partir del Informe n. 33 de Análisis de los casos de COVID-19 notificados a la RENAVE hasta el 10 de mayo en España a 29 de mayo de 2020 y se reanudaron a partir del 15 de julio con el informe n. ${ }^{\circ} 34$ que recoge los datos en función de la nueva Estrategia de Vigilancia y Control. Los informes a partir de ese momento cambiaron su estructura y ya no recogen datos relacionados con las características de las personas fallecidas, salvo aquellos relacionados con la edad, que continúan mostrando unos valores muy superiores en personas a partir de 70 y 80 años en relación a la necesidad de hospitalización y al número de personas fallecidas. 
3. El derecho a la salud de las personas con discapacidad intelectual o del desarrollo en situaciones de emergencia

Todos los seres humanos nacemos libres en dignidad y derechos según el artículo 1 de la Declaración Universal de los Derechos Humanos (1948). La dignidad es un valor intrínseco a todo ser humano y todos los derechos humanos derivan de ella.

La esencia de lo humano se asienta en la consideración de cada persona como portadora de valor en sí misma. La persona lo es; sin más, siempre y en toda circunstancia y nunca deja de serlo, ni parcialmente, por el hecho de disponer de mayor o menor competencia de ningún tipo o de mayor o menor poder, sea de la clase que sea. [ ] El reconocimiento de la persona con discapacidad intelectual es condición imprescindible para hacer realidad la dignidad (FEAPS, 2006).

Las Naciones Unidas (2000), en su Observación General 14, reconocen a todo ser humano el derecho al más alto nivel de salud; incluso en situaciones de graves limitaciones de recursos, es preciso proteger a los miembros vulnerables de la sociedad. La Convención sobre los Derechos de las Personas con Discapacidad hace explícito que las personas con discapacidad tienen derecho a disfrutar de este derecho sin discriminación y que los Estados Parte que la han ratificado deben impedir que se nieguen, de manera discriminatoria, servicios de salud o atención a la salud. Una diferencia de trato únicamente podrá resultar no discriminatoria cuando se pueda justificar en criterios razonables y objetivos.

España, con la firma de la Convención, reconoce que la discapacidad forma parte de la condición humana y que la deficiencia no es un motivo legítimo para restringir derechos humanos. Esto supone la superación del modelo que, como señalan las $\mathrm{Na}-$ ciones Unidas (2018), "no reconoce a las personas con discapacidad como titulares de derechos, sino que estas quedan reducidas a sus deficiencias" y "las deficiencias se consideran un motivo legítimo para restringir o denegar derechos”.

El derecho a la salud implica que todas las personas, independientemente de sus condiciones personales, tienen derecho a tratamiento, lo que "comprende la creación de un sistema de atención médica urgente en los casos de accidentes, epidemias y peligros análogos para la salud, así como la prestación de socorro en casos de desastre y de ayuda humanitaria en situaciones de emergencia” (Naciones Unidas, 2000).

Las situaciones de riesgo y emergencia humanitaria, como la derivada de la enfermedad causada por la infección del SARS-CoV-2, aparecen reguladas en el artículo 11 de la Convención sobre los derechos de las personas con discapacidad (Naciones Unidas, 2006), estableciéndose que los Estados están obligados a tomar todas las medidas necesarias para garantizar la seguridad y protección de las personas con discapacidad en situaciones de riesgo.

Por último, es preciso tener en cuenta que ningún derecho es absoluto y

situar la gestión y la recuperación de la pandemia en el discurso de los derechos no supone encontrar la solución precisa para cada problema que se plantee. Lo normal será (esto es lo habitual en la vida de los derechos) encontrarse con situaciones en las que la toma de decisión se realiza en un contexto de colisión entre derechos, es decir, hay dos 
derechos enfrentados o un mismo derecho a satisfacer para distintas personas. Se trata de elegir y ponderar, tomando una decisión que, aunque pueda afectar a un derecho, sea justificable desde el propio discurso de los derechos. (De Asís, 2020)

\section{Distribución justa de los recursos sanitarios en momentos de crisis}

Tal y como se ha puesto de relieve anteriormente, en las etapas de mayor incidencia de la enfermedad COVID-19 se ha producido escasez de algunos recursos sanitarios, algo que también se señala en el trabajo de Emmanuel et al. (2020), empezando por los tests diagnósticos y siguiendo por las camas de hospitalización general, las camas de UCI y equipamientos especializados como ventiladores. En algunas ocasiones se ha visto afectada la disponibilidad de profesionales sanitarios, ya que muchos se han infectado por el virus teniendo que mantenerse en cuarentena sin poder acudir a sus puestos de trabajo o convirtiéndose ellos mismos en enfermos demandantes de asistencia. Incluso en momentos puntuales se ha producido escasez de ciertos medicamentos destinados bien a tratamientos experimentales bien a paliar los síntomas de la enfermedad.

Esta escasez de recursos ha obligado a que, en los momentos de mayor presión asistencial, haya sido necesario proceder a priorizar la atención de unos pacientes sobre otros. Es importante recordar que este proceso de asignación prioritaria de los recursos sanitarios según las necesidades de los pacientes no es una novedad ni una consecuencia de la pandemia, sino un procedimiento habitual de cualquier sistema de salud que puede apreciarse en la existencia de listas de espera o de triajes en los servicios de urgencias.

La peculiaridad de la situación generada por la COVID-19 es que, ante la elevada probabilidad de colapso del sistema sanitario, ha sido preciso tomar gran cantidad de decisiones relacionadas con la priorización de pacientes en un corto espacio de tiempo. Decisiones que han generado consecuencias, en ocasiones muy graves, sobre la salud de muchos pacientes e incluso la muerte de algunos de ellos, y que se han visto obligados a tomar profesionales estresados, en momentos de máxima presión y, quizá, sin los apoyos necesarios.

Ante la posibilidad de que nuevos repuntes de la enfermedad nos sitúen de nuevo en la necesidad de tomar más decisiones de priorización de la asistencia sanitaria, es imprescindible reflexionar sobre los principios que deben guiar esta toma de decisiones tanto en lo referente a la población general como en lo referente al colectivo de personas con DID.

Tal y como plantean Emmanuel et al. (2020), "La pregunta no es si establecer prioridades, sino cómo hacerlo de manera ética y consistente, antes de basar las decisiones en enfoques de instituciones individuales o en la intuición de un clínico en el calor del momento”. De manera general, ¿cuáles deben ser los principios que guíen la priorización de los recursos sanitarios en situaciones en las que son escasos?

Emmanuel et al. (2020) plantean cuatro valores sobre los que priorizar los recursos, a saber: maximizar los beneficios producidos por los escasos recursos, tratar a 
todas las personas igualmente, promocionar y premiar el valor instrumental y dar prioridad a las personas más vulnerables.

A partir de estos valores los autores plantean seis recomendaciones específicas para la asignación de recursos sanitarios en la pandemia de COVID-19:

Recomendación 1: priorizar el tratamiento de las personas que están enfermas y podrían recuperarse sobre aquellos que es poco probable que se recuperen incluso si reciben tratamiento y aquellos que es probable que se recuperen sin tratamiento.

Recomendación 2: las intervenciones críticas relacionadas con la COVID-19; pruebas diagnósticas, equipos de protección individual, camas de UCI, ventiladores, terapias y vacunas deben ir primero a trabajadores de atención sanitaria de primera línea y otros que atienden a pacientes enfermos y que mantienen en funcionamiento infraestructuras críticas, particularmente trabajadores que se enfrentan a un alto riesgo de infección y cuyas competencias hacen difícil su reemplazo.

Recomendación 3: para pacientes con pronósticos similares, la igualdad debe ser invocada y operacionalizada a través de asignación aleatoria, como una lotería, en lugar de seguir un proceso de asignación del tipo el primero que llega es el primero en ser atendido. Debe recordarse que primar la atención de aquellos que llegan antes a solicitarla puede producir situaciones paradójicas como beneficiar a unos ciudadanos sobre otros por el simple hecho de vivir más cerca de un centro sanitario o primar la atención de aquellos que, por no seguir las recomendaciones sanitarias, enferman antes perjudicando a quienes, respetando las normas, terminan enfermando después.

Recomendación 4: las pautas de priorización deben diferir según la intervención y responder a la evidencia científica cambiante.

Recomendación 5: aquellas personas que participan o han participado en investigaciones para demostrar la seguridad y efectividad de las vacunas y los diferentes tratamientos deberían recibir alguna prioridad para las intervenciones relacionadas con la COVID-19.

Recomendación 6: no debería haber diferencia en la asignación de recursos escasos entre pacientes con COVID-19 y aquellos con otras enfermedades.

Por su parte, Warrillow et al. (2020), en su redacción de la guía para la toma de decisiones complejas durante la pandemia de COVID-19 de la Australian and New Zealand Intensive Care Society (ANZICS), se centraron principalmente en el acceso a las Unidades de Cuidados Intensivos.

Warrillow et al. (2020) plantean que las decisiones deben tomarse de manera justa, consistente, transparente y de acuerdo con una política que debe llevar a que esto sea posible. Más que la asignación de recursos basada únicamente sobre lo que podría beneficiar a pacientes individuales, habrá una necesidad de posicionar la asignación de recursos teniendo en mente el bien de la comunidad en su conjunto, de tal forma que se derive el máximo beneficio para todos con los recursos disponibles.

La guía de ANZICS reconoce que, durante los picos máximos de incidencia de la pandemia, algunos pacientes pueden morir como resultado de la escasez de recursos y que esta situación puede ocurrir incluso con la planificación y la coordinación óptimás. Una aproximación consecuente que asegure el mayor beneficio y el menor daño para el máximo número de personas está justificada; sin embargo, pueden incorporarse 
algunas protecciones que aseguren que los grupos en situación de vulnerabilidad no quedan desamparados y el principio de igualdad para todos se mantenga. La guía señala que el uso único de exclusiones categóricas de poblaciones específicas de pacientes no es recomendable. Warrillow et al. (2020) recomiendan seguir los siguientes principios para la toma de decisiones:

Todos los pacientes, incluidos niños y adultos con y sin infección por SARSCoV-2, que estén siendo tratados en una UCI, deben recibir una consideración justa de sus necesidades de atención de cuidados intensivos y un acceso justo a ellos.

La priorización clínica debe ser la estrategia inicial para determinar el acceso a los cuidados intensivos cuando los recursos son limitados. La valoración completa del paciente debería incluir debates sobre los objetivos de cuidado, las preferencias del paciente y la familia y la aceptación por el paciente de las intervenciones de cuidados críticos si se le ofrecen. Los cuidadores y familiares de pacientes adultos incapaces de comunicar sus preferencias de manera efectiva pueden (y deben) proporcionar percepciones muy valiosas sobre sus deseos.

En la situación en la que algunos pacientes estén igualados en términos de prioridad clínica, el acceso a cuidados intensivos no debe estar basado en consideraciones irrelevantes y discriminatorias como género, orientación sexual, religión, discapacidad, estatus social, contactos personales, riqueza, ciudadanía, aseguramiento, etnia o raza. En estos casos puede ser éticamente justificable considerar otros determinantes para la priorización incluyendo, aunque no exclusivamente:

- El apoyo a pacientes pertenecientes a grupos sujetos a deprivación y desventaja social como medio de reparación de su situación de vulnerabilidad.

- Considerar la priorización de adultos con responsabilidades de cuidado (cargas familiares).

- Abogar por que los pacientes más jóvenes que han vivido pocas etapas de vida tengan prioridad sobre los pacientes mayores.

- Apoyar a las personas que están en la primera línea de cuidado y están expuestos a riesgo de infección debido a las actividades inherentes a su puesto.

- En algunos casos el tratamiento intensivo no proporciona el beneficio que se esperaba o un paciente puede desarrollar complicaciones y su supervivencia es improbable. En esas circunstancias difíciles es justificable considerar el cese de la terapia intensiva.

- Es apropiado considerar un traslado temprano de los pacientes que mejoren a plantas de hospitalización general cuando los recursos son escasos, si esto crea capacidad para admitir pacientes adicionales con grandes necesidades.

Una guía similar a la de ANZICS fue publicada en España por el Grupo de Trabajo de Bioética de la Sociedad Española de Medicina Intensiva, Crítica y Unidades Coronarias (SEMICYUC) (2020) bajo el título de Recomendaciones éticas para la toma de decisiones en la situación excepcional de crisis por pandemia COVID-19 en las unidades de cuidados intensivos. 
Para SEMICYUC (2020) los principios claves en gestión de situaciones de atención en crisis han de ser justicia, deber de cuidar, deber de administrar recursos, transparencia, consistencia, proporcionalidad y responsabilidad.

El documento señala que en el caso de desproporción entre la demanda y las posibilidades de atención sería lícito establecer un triaje de ingreso entre los pacientes, basado en el principio de justicia distributiva, evitando seguir el criterio habitual de "el primero en llegar es el primero en recibir asistencia”. Realizan 24 recomendaciones generales y 11 recomendaciones específicas para la toma de decisiones en la situación excepcional de la pandemia COVID-19. Seleccionamos a continuación las más relevantes para el objetivo de este trabajo eliminando fundamentalmente las de carácter más técnico relacionadas con la especificidad de la medicina intensiva.

Recomendaciones generales a tener en cuenta en la toma de decisiones en la asignación de recursos limitados según SEMICYUC:

- Siempre debe existir un beneficio grande esperable y reversibilidad.

- Aplicar criterios estrictos de ingreso en UCI basados en maximizar el beneficio del bien común.

- En caso de complicaciones, o prevista mala evolución tanto clínica como funcional, plantear la retirada terapéutica sin dilación por futilidad ${ }^{2}$ e iniciar la aplicación de medidas paliativas.

- Se debe valorar el paciente de forma global y no la enfermedad de forma aislada.

- Ante dos pacientes similares, se debe priorizar a la persona con más años de vida ajustados a la calidad. Priorizar la mayor esperanza de vida con calidad.

- En personas mayores se debe tener en cuenta la supervivencia libre de discapacidad por encima de la supervivencia aislada.

- Tener en cuenta otros factores como, por ejemplo, personas a cargo del paciente para tomar decisiones maximizando el beneficio del máximo de personas.

- Tener en cuenta el valor social de la persona enferma.

- Contactar con el Comité de Ética Asistencial para que pueda ayudar en la prevención o resolución de conflictos de valores entre las partes implicadas (profesionales, pacientes, familiares e institución) relacionados con la toma de decisiones.

Recomendaciones específicas a tener en cuenta en la toma de decisiones en la asignación de recursos limitados:

- Cualquier paciente con deterioro cognitivo, por demencia u otras enfermedades degenerativas, no sería subsidiario de ventilación mecánica invasiva.

- El criterio médico en cada paciente está por encima de estas recomendaciones generales, siempre que sea razonado, argumentado y se consensúe en la sesión clínica diaria o por el Comité de Ética Asistencial.

2 El concepto de futilidad en medicina hace referencia a la cualidad de una actuación médica que o bien es inútil para conseguir un objetivo beneficioso o bien lo consigue, pero a costa de molestias o sufrimientos desproporcionados para el paciente o a costa de serios inconvenientes económicos, familiares o sociales. Las acciones médicas fútiles no deben iniciarse. 
- Las decisiones de adecuación terapéutica, idealmente, deben consensuarse con el paciente y/o familiares, si no hay posibilidad de consensuar las decisiones, recordar que el garante de la toma de decisiones es el equipo sanitario del paciente.

Por su parte, la Comisión Central de Deontología del Consejo General de Colegios Oficiales de Médicos (españoles) (CGCOM) (2020) realizó un informe en el que recoge consideraciones éticas y deontológicas ante la limitación del acceso a la atención sanitaria a los pacientes. En este informe indica que todos los enfermos que lo precisen deben ser atendidos de la mejor manera posible en un Sistema Nacional de Salud (SNS) "que es de todos y para todos".

La Comisión Central de Deontología señala que la valoración deontológica de cualquier priorización para el ingreso y atención a pacientes graves y críticos en situaciones de limitación de recursos requiere realizar, entre otras, las siguientes consideraciones:

- El criterio fundamental debe estar basado en las probabilidades de supervivencia de cada paciente.

- No pueden ser criterios de priorización ni el orden de petición de asistencia, ni el de llegada a los servicios de urgencias hospitalarias, ni solo la edad de los pacientes.

- No en todas las situaciones clínicas o muy avanzadas y con carácter irreversible se debe proceder al ingreso hospitalario.

- Las tomas de decisiones de tanta relevancia deben ser individuales y personales, porque, aunque los procesos puedan coincidir, cada paciente puede ser distinto. El conocimiento científico es imprescindible, pero no basta para ayudar a los pacientes a curarse o a convivir con sus enfermedades lo mejor posible.

\section{Distribución justa de recursos sanitarios en una crisis y personas con DID}

Tomar en consideración las especiales características del colectivo de personas con DID en las circunstancias relacionadas con la limitación de acceso a los servicios sanitarios en situaciones de emergencia es de especial relevancia por diferentes motivos.

En primer lugar, existe una amplia bibliografía que acredita que, de manera general, las personas con DID tienen un peor estado de salud en comparación con la población general, que es un colectivo que recibe una asistencia sanitaria de peor calidad que está condicionando la aparición de desigualdades como barreras de acceso a los servicios sanitarios (Muñoz y Marín 2005; Martínez Leal et al. 2011) y un elevado número de muertes evitables definidas según Gisopert et al. (2006) como "los casos de muerte por procesos que disponen de tratamiento o medidas de prevención y que podrían haberse evitado si el sistema sanitario hubiera actuado correctamente en todos sus pasos".

En segundo lugar, la experiencia en situaciones en las que debe decidirse sobre si aplicar o no un tratamiento a una persona con DID, como son las relativas a la consideración de futilidad de una intervención médica, no es muy positiva para el 
colectivo. Tal y como señala el National Council on Disability de EE. UU. (2019), existen sesgos negativos y supuestos inexactos sobre la calidad de vida de las personas con discapacidad que son dominantes en la sociedad, y de las que los sanitarios no están exentos, que pueden derivar en la toma de decisiones discriminatorias sobre el colectivo con el resultado de la devaluación o negación de algunos tratamientos.

Las decisiones de futilidad médica a menudo carecen de objetividad y garantías procesales, dejando espacio para que los profesionales sanitarios hagan recomendaciones impactados por sesgos negativos sobre la calidad de vida de las personas con discapacidad y lo mismo puede llegar a ocurrir en situaciones de emergencia y escasez de recursos sanitarios.

El National Council on Disability (2019) señala que ha sido bien documentado que los proveedores de atención médica tienden a infravalorar significativamente la vida con discapacidad. Como resultado, estos responsables de la atención sanitaria siguen sin ser conscientes de la alta calidad de vida y felicidad que experimentan muchas personas con discapacidad. Esta falta de conciencia ha impactado sobre la toma de decisiones de futilidad médica y, en algunos casos, ha robado y roba a las personas con discapacidad la oportunidad de recuperarse de algunas enfermedades.

En tercer lugar, existen posiciones de algunos profesionales sanitarios plasmadas en publicaciones científicas en las que se asume que, en situaciones de emergencia sanitaria como la generada por la COVID-19, las personas con DID no son tristemente elegibles para ser tratadas en UCI (Arango, 2020).

Por tanto, resulta imprescindible realizar algunas consideraciones específicas sobre la priorización de recursos sanitarios escasos en situaciones de crisis y discapacidad en general y DID en particular.

A este respecto el Comité de Bioética de España (CBE) (2020) emitió un informe acerca de las implicaciones éticas que para las personas con discapacidad pueden tener las Recomendaciones éticas para la toma de decisiones en la situación excepcional de crisis por pandemia COVID-19 en las unidades de cuidados intensivos realizadas por la SEMICYUC a las que hemos hecho referencia anteriormente.

En su informe el CBE recuerda, citando otro informe previo (CBE, 2008), que, en lo relativo a la asignación de recursos sanitarios escasos, la bioética está plagada de este tipo de elecciones trágicas donde cualquier decisión sobre la distribución de recursos afecta de manera sustancial a la vida de las personas, pues la elección no resulta ser entre un mal y un bien, sino entre dos males, y avisa de que puede caerse en el error de primar casi exclusivamente el interés colectivo en detrimento de la dignidad y derechos del individuo.

Se plantea, por tanto, que habría que adoptar unos criterios en la asignación de recursos que sean comunes para todos los ciudadanos, de modo que no se produzcan graves inequidades asistenciales entre unos y otros. Cualquier criterio que se adopte deberá basarse en el pleno respeto a la dignidad de la persona, la equidad y la protección frente a situaciones de vulnerabilidad.

Con relación a las recomendaciones de SEMICYUC (2020), el CBE señala que las propuestas llevadas a cabo por sociedades científicas y otros grupos de trabajo son bienvenidas, más aún cuando vienen presuntamente avaladas por la mejor evidencia 
científica que se deriva de la propia cualificación profesional de quienes las están emitiendo. Sin embargo, en un contexto de escasez de recursos donde se trata de limitar el derecho a la protección de la salud de algunos individuos, la decisión solo puede corresponder única y exclusivamente a la autoridad pública, la única facultada constitucionalmente para limitar y suspender derechos.

Continúa señalando el informe del CBE que, cuando en el contexto de la crisis del coronavirus se habla de priorizar, se está haciendo referencia a limitar o suspender el derecho a la protección de la salud reflejado en el artículo 43 de la Constitución española (1978). Las sociedades científicas, o los comités de expertos, son fundamentales para proveer a la autoridad pública de los conocimientos necesarios para establecer unos criterios nacionales de priorización, pero en modo alguno constituyen las entidades adecuadas para fijar dicha priorización. Así lo señala también la United Nations Human Rights office of the High Commissioner (2020) en su guía sobre COVID-19 y los derechos de las personas con discapacidad, en la que indica que algunas pautas y protocolos médicos selectivos pueden aumentar la discriminación a la que se enfrentan las personas con discapacidad en la prestación de servicios de salud. Estos protocolos a veces revelan prejuicios médicos contra las personas con discapacidad con respecto a su calidad de vida y valor social. Este punto relativo a las guías y recomendaciones de las sociedades científicas y grupos de expertos es de especial relevancia, ya que son muy comunes los artículos científicos en los que se orienta a los profesionales sanitarios a emplear como criterios de inclusión/exclusión de pacientes, en según qué tratamientos, este tipo de recomendaciones (Rascado Sedes et al., 2020).

Con relación a las personas con discapacidad el CBE afirma que, si bien en un contexto de recursos escasos se puede justificar la adopción de un criterio de asignación basado en la capacidad de recuperación del paciente, en todo caso se debe prevenir la extensión de una mentalidad utilitarista o, peor aún, de prejuicios contrarios hacia las personas mayores o con discapacidad. El término "utilidad social” que aparece en alguna de las recomendaciones publicadas parece extremadamente ambiguo y éticamente discutible, porque todo ser humano por el mero hecho de serlo es socialmente útil en atención al propio valor ontológico de la dignidad humana. Conceptos tales como "utilidad social" o similares no responden a los principios bioéticos que deben informar la toma de decisiones en el ámbito asistencial.

Con relación a las recomendaciones de SEMICYUC (2020), el CBE insiste en considerar que algunos de los conceptos que se emplean en las mismas, sobre todo, los que hacen referencia a cuestiones tales como "supervivencia libre de discapacidad por encima de la supervivencia aislada" o la que recomienda que "cualquier paciente con deterioro cognitivo, por demencia u otras enfermedades degenerativas, no serían subsidiarios de ventilación mecánica invasiva”, no son compatibles con ningún Tratado Internacional de Derechos Humanos y, especialmente, con la Convención sobre los Derechos de las Personas con Discapacidad.

Las decisiones sobre la asignación de recursos médicos escasos no pueden tomarse en función de criterios no médicos como edad o condición de discapacidad, ya que ello supone una discriminación y vulneraría la Convención sobre los Derechos de las Personas con Discapacidad que exige garantizar el derecho a la vida y la atención sanitaria 
a las personas con discapacidad en igualdad de condiciones con todas las demás. Lesionaría, además de forma flagrante, principios éticos elementales, pues supondría entender que la vida de las personas con discapacidad tiene menos calidad y, por tanto, merece menos la pena atenderla, lo que resulta no solo absolutamente incoherente con la visión de la discapacidad que hoy tiene la sociedad española, sino que supondría establecer una división entre vidas humanas en función de un supuesto valor de esas vidas arbitrariamente asignado o en función de su utilidad social.

El CBE señala que la toma de decisiones de priorización de recursos ha de ser necesariamente individualizada, aunque se adopten siguiendo los criterios establecidos por protocolos y recomendaciones como soporte técnico-científico. Los profesionales deben contar con la participación de los correspondientes Comités de Etica Asistencial que ya están implantados en la mayoría de los hospitales. Estando completamente de acuerdo con esta afirmación y siendo los Comités de Ética Asistencial un instrumento fundamental, no debe olvidarse que, tal y como señala el National Council on Disability (2019), estos comités internos de ética pueden no ser un foro ideal para mediar y tomar decisiones de este tipo ya que, siendo como son un mecanismo propio de cada hospital, pueden estar sujetos a conflictos de intereses financieros, profesionales y personales y/o a presiones políticas especialmente intensas en situaciones de emergencia. Además, la composición de los comités de ética asistencial de los hospitales rara vez refleja la diversidad social, por ejemplo, racial, étnica, religiosa o relacionada con la discapacidad, por lo que sus decisiones pueden verse influenciadas por sesgos negativos sobre algunos de esos colectivos.

\section{Conclusiones}

- El derecho a la salud sin discriminación es un derecho fundamental que no puede verse vulnerado ni siquiera en situaciones de escasez de recursos

El derecho a la salud es un derecho humano fundamental, recogido en diversos Tratados Internacionales de Derechos Humanos, que deriva de la dignidad inherente de las personas y que obliga a los Estados a generar condiciones para que todos sus ciudadanos aspiren al más alto nivel de salud posible.

Por ello, los Estados están obligados a proteger el derecho a la salud de todos los ciudadanos, adoptando medidas positivas para darle plena efectividad. Debe garantizarse que existirá un número suficiente de establecimientos, bienes y servicios públicos de atención sanitaria accesibles a todos sin discriminación y que sean respetuosos con la ética médica. Todas las personas, incluidas las personas con discapacidad, cuando están enfermas tienen derecho a exigir a los poderes públicos asistencia sanitaria y a que esta asistencia que se les preste sea técnicamente correcta.

Es evidente que en una pandemia especialmente grave como la de la COVID-19, con capacidad para saturar los servicios sanitarios, resulta imprescindible y justificado establecer protocolos de priorización de la asignación de los recursos disponibles. Debe asumirse además que, en los picos máximos de incidencia de una pandemia de 
este tipo, algunos pacientes pueden morir debido a la insuficiencia de recursos. No obstante, el respeto a los derechos humanos y, en concreto, el derecho a la salud sin discriminación suponen un límite que es imprescindible respetar en cualquier toma de decisiones.

- La asignación de recursos deberá realizarse estableciendo criterios neutros con la discapacidad y basados en el bien de la comunidad en su conjunto, de forma que se derive el máximo beneficio para todos

Parece existir una coincidencia generalizada sobre algunos criterios básicos, como que debe priorizarse a algunos colectivos como los profesionales sanitarios o aquellos otros profesionales de los que dependen infraestructuras esenciales o que, en ningún caso, debe aplicarse únicamente el criterio de atender primero al que primero acude a solicitar asistencia. Sin embargo, debe garantizarse para todos los ciudadanos y más concretamente para las personas con discapacidad que, en esta llamada al bien común, no se generen situaciones de discriminación en las que reciban un trato menos favorable que otras personas por razón de su discapacidad. Ningún criterio o recomendación para la priorización de la asistencia sanitaria podrá ser considerado conforme a derecho si se realiza sobre la base de una discriminación.

- Es preciso aplicar dichos criterios de priorización evitando la influencia de los estereotipos y prejuicios imperantes sobre la discapacidad

A la hora de realizar los juicios de valor necesarios para aplicar criterios de priorización es esencial evitar que los estereotipos y prejuicios hacia la discapacidad sesguen la valoración que realicen del paciente los profesionales sanitarios y, para ello, el Estado debe garantizar que estos profesionales cuentan con un conocimiento preciso de la discapacidad. También las propias personas con discapacidad o sus organizaciones representativas deberían estar incluidas en los órganos que asesoran en el establecimiento de los criterios de priorización asistencial para evitar estos sesgos.

Cualquier priorización basada en evaluaciones de la calidad de vida del paciente deberá evitar realizarse sobre la consideración de que la calidad de vida de una persona con discapacidad, y más concretamente con DID, es menor debido a la presencia de la propia discapacidad. Esto no es necesariamente así: hay personas con DID con una excelente calidad de vida y, por el contrario, pueden encontrarse muchas personas sin DID con mala calidad de vida.

Debe ser plenamente aceptado el hecho de que la presencia de una discapacidad, y más concretamente la de una discapacidad intelectual o del desarrollo, per se, no limita la supervivencia de las personas tras contraer la COVID-19. La discapacidad intelectual o del desarrollo no implica una situación de terminalidad y en ningún caso puede servir para justificar la denegación de la asistencia sanitaria o la limitación del esfuerzo terapéutico. Lo contrario resulta inadmisible, ya que implica partir de la 
consideración de que las vidas de las personas con discapacidad tienen menor valor que las del resto de ciudadanos.

Asimismo, cualquier criterio de priorización que se establezca en la atención sanitaria deberá ser neutro con la discapacidad, es decir, deberá ser aplicable por igual a personas con y sin discapacidad. La utilización de un criterio de priorización del tipo "supervivencia libre de discapacidad", aun cuando vaya referido a personas ancianas, resulta innecesario y supone en sí mismo un juicio de valor negativo sobre la discapacidad como algo indeseable que evoca planteamientos eugenésicos. Del mismo modo, la utilización del criterio del "valor social" del paciente, aparte de ser imposible de evaluar de manera objetiva, resulta contrario, como ya se ha establecido, a la dignidad inherente y al valor intrínseco de las personas.

\section{- La toma final de decisiones siempre debe ser en función de cada persona concreta}

Tomar decisiones de tanta relevancia debe hacerse de manera individual y personal, porque, aunque los procesos y los momentos de la enfermedad puedan coincidir, cada paciente es distinto. Debe huirse del error de primar el interés colectivo en detrimento de la dignidad y derechos del individuo.

Tomar en consideración las reflexiones anteriores es fundamental no solo para su aplicación durante los picos pandémicos, sino también en el marco de la hospitalización del paciente con discapacidad. Es esencial considerarlas ante la toma de decisiones en otras situaciones como la de aplicar tests diagnósticos a las personas con DID que presenten síntomas; garantizar su bienestar durante los posibles periodos de cuarentena, tanto en sus hogares como en servicios de atención residencial; asegurar su acceso a las posibles medicaciones específicas para la enfermedad y/o a las vacunas, o promocionar investigaciones específicas sobre el impacto de la enfermedad sobre el colectivo.

\section{Referencias bibliográficas}

Arango, C. (2020). Lessons learned from the Coronavirus health crisis in Madrid, Spain: how COVID-19 has changed our lives in the last 2 weeks. Biological Psychiatry, 88(7), e33-e34. https://doi.org/10.1016/j.biopsych.2020.04.003

Asamblea General. (1948). Declaración Universal de los Derechos Humanos. Naciones Unidas.

Asociación Americana de Discapacidades Intelectuales y del Desarrollo (AAIDD). (2011). Discapacidad intelectual: definición, clasificación y sistemas de apoyo. 11. edición. Alianza Editorial.

Centro de Coordinación de Alerta y Emergencias (CCAES). (s. f.). Alertas en salud pública de actualidad. Enfermedad por nuevo coronavirus, COVID-19. Recuperado el 10 de junio de 2020 en https://www.mscbs.gob.es/profesionales/saludPublica/ccayes/alertasActual/nCov-China/situacionActual.htm 
REFLEXIONES SOBRE EL ACCESO A LOS SERVICIOS DE SALUD DE LAS PERSONAS CON DISCAPACIDAD INTELECTUAL O DEL DESARROLLO EN EL MARCO DE LA CRISIS SANITARIA CREADA POR LA PANDEMIA DE LA COVID-I9 J. MUÑOZ E I. DE ARAOZ

Comité de Bioética de España. (2017). Informe sobre la financiación pública del medicamento profilaxis pre-exposición (PrEP) en la prevención del VIH. Recuperado el 1 de junio de 2020 en http://assets.comitedebioetica.es/files/documentacion/es/Informe_PrEP.pdf

Comité de Bioética de España. (2020). Informe del Comité de Bioética de España sobre los aspectos bioéticos de la priorización de recursos sanitarios en el contexto de la crisis del coronavirus. Recuperado el 1 de junio de 2020 en http://assets.comitedebioetica.es/files/ documentacion/Informe $\% 20 \mathrm{CBE}$ - $\% 20$ Priorizacion $\% 20 \mathrm{de} \% 20$ recursos $\% 20$ sanitarioscoronavirus $\% 20 \mathrm{CBE}$.pdf

Consejo General de Colegios Oficiales de Médicos de España. (2020). Informe de la Comisión Central de Deontología en relación a la priorización de las decisiones sobre los enfermos en estado crítico en una catástrofe sanitaria. Recuperado el 3 mayo de 2020 en https:// comsalamanca.es/publi/2020/FOR20/200323_priorizacion_decisiones_enfermos.pdf

Constitución española. (1978). Boletín Oficial del Estado, 29 de diciembre de 1978, 311.

De Asís, R. (2020). Recordando lo obvio. Maga3ine. https://uc3m-magazine.uc3m. es/2020/05/12/recordando-lo-obvio/

Emanuel, E. J., Persad, G., Upshur, R., Thome, B., Parker, M., Glickman, A., Zhang, C., Boyle, C., Smith, M. y Phillips, J. P. (2020). Fair allocation of scarce medical resources in the time of Covid-19. The New England Journal of Medicine, 382(21), 2049-2055. https:// doi.org/10.1056/NEJMsb2005114

FEAPS. (2006). Código ético. Recuperado el 15 de junio de 2020 en https://sid.usal.es/idocs/ F8/FDO6670/codigo_etico.pdf

Fernández Bretón, E. (2020). Obesidad. Revista Española de Salud Pública. Recuperado el 29 de diciembre de 2020 en https://www.mscbs.gob.es/biblioPublic/publicaciones/recursos_propios/resp/revista_cdrom/Suplementos/Pildoras/pildora14_obesidad.pdf

Folch, A., Salvador-Carulla, L., Vicens, P., Cortés, M. J., Irazábal, M., Muñoz, S., Rovira, L., Orejuela, C., González, J. A. y Martínez-Leal, R. (2019). Health indicators in intellectual developmental disorders: the key findings of the POMONA-ESP project. Journal of Applied Research in Intellectual Disabilities, 32(1), 23-34.

Gispert, R., Arán Barés, M. D. y Puigdefàbregas, A. (2006). La mortalidad evitable: lista de consenso para la actualización del indicador en España. Gaceta Sanitaria, 20(3), 184-193.

Johns Hopkins University. (s. f.). COVID-19 Dashboard by the Center for Systems Science and Engineering (CSSE). Recuperado el 10 de junio de 2020. https://coronavirus.jhu.edu/ map.html

Martínez-Leal, R., Salvador-Carulla, L., Gutiérrez-Colosía, M. R., Nadal, M., Novell-Alsina, R., Martorell, A., González-Gordón, R. G., Mérida-Gutiérrez, M. R., Ángel, S., Milagrosa-Tejonero, L., Rodríguez, A., García-Gutiérrez, J. C., Pérez-Vicente, A., García-Ibáñez, J. y Aguilera-Inés, F. (2011). La salud en personas con discapacidad intelectual en España: estudio europeo POMONA-II [Health among persons with intellectual disability in Spain: the European POMONA-II study]. Revista de Neurología, 53(7), 406-414.

Ministerio de Sanidad. (s. f.). Información cientifica-técnica. Enfermedad por coronavirus, COVID-19. Recuperado el 2 de junio 2020 en https://www.mscbs.gob.es/profesionales/ saludPublica/ccayes/alertasActual/nCov-China/documentos/ITCoronavirus.pdf

Muñoz, J., Fernández, A. B., Belinchón, M., Sellán, C., Díaz, M. L., Hernando, M. F. y MuÑoz, S. (2014). Discapacidad intelectual y salud: derechos, desigualdades, evidencias y propuestas. Recuperado el 29 de diciembre de 2020 en https://www.plenainclusion.org/ sites/default/files/discapacidad_intelectual_y_salud.pdf 
REFLEXIONES SOBRE EL ACCESO A LOS SERVICIOS DE SALUD DE LAS PERSONAS CON DISCAPACIDAD INTELECTUAL O DEL DESARROLLO EN EL MARCO DE LA CRISIS SANITARIA CREADA POR LA PANDEMIA DE LA COVID-I9 J. MUÑOZ E I. DE ARAOZ

Muñoz, J. y Marín, M. (2005). Necesidades sanitarias de las personas con discapacidad intelectual y sus familias. Siglo Cero, 36, 215, 5-24.

Naciones Unidas. (2000). El derecho al disfrute del más alto nivel posible de salud. Recuperado el 21 de junio de 2020 en https://www.acnur.org/fileadmin/Documentos/BDL/2001/1451. pdf?file=fileadmin $/$ Documentos $/$ BDL $/ 2001 / 1451$

Naciones Unidas. (2006). Convención sobre los Derechos de las Personas con Discapacidad. Recuperado el 21 de junio de 2020 en https://www.un.org/esa/socdev/enable/documents/ tccoonvs.pdf

Naciones Unidas. (2018). Observación general núm. 6 (2018) sobre la igualdad y la no discriminación. Recuperado el 21 de junio de 2020 en http://docstore.ohchr.org/SelfServices/ FilesHandler.ashx? enc $=6$ QkG1d\%2fPPRiCAqhKb7yhsnbHatvuFkZ\%2bt93Y3D\%2ba a2qtJucAYDOCLUtyUf\%2brfiOZ88SbKi18LECUG89QSdTKcQfnbxosDFSIVZSIPG GT7aQ9xSV9ZM3t763zmWeZKYHI

National Council On Disability. (2019). Medical futility and disability bias: part of the bioethics and disability series. Recuperado el 30 de abril de 2020 en https://ncd.gov/sites/ default/files/NCD_Medical_Futility_Report_508.pdf

Organización Mundial de la SAlud. (s. f.). Investigaciones epidemiolo?gicas y clínicas precoces sobre el COVID-19 para una respuesta de salud pública. Recuperado el 19 de junio de 2020 en https://www.who.int/docs/default-source/coronaviruse/200223-early-investigations-one-pager-v2-spanish.pdf?sfvrsn=8aa0856_14

Rascado Sedes, P., Ballesteros Sanz, M. A., Bodí Saera, M. A., Carrasco RodríguezRey, L. F., Castellanos Ortega, A., Catalán González, M., López, C. H., Díaz Santos, E., Escriba Barcena, A., Frade Mera, M. J., Igeño Cano, J. C., Martín Delgado, M. C., Martínez Estalella, G., Raimondi, N., Roca i Gas, O., Rodríguez Oviedo, A., Romero San Pío, E., Trenado Álvarez, J., Junta directiva de la SEMiCYUC y JunTA diReCTIVA DE LA SEEIUC (2020). Contingency plan for the intensive care services for the COVID-19 pandemic. Plan de contingencia para los servicios de medicina intensiva frente a la pandemia COVID-19. Medicina Intensiva, S0210-5691(20)30095-4. Advance online publication. https://doi.org/10.1016/j.medin.2020.03.006

RENAVE, CNE, CNM (ISCIII). (2020). Informes COVID-19. Recuperado el 19 de junio de 2020 en https://www.isciii.es/QueHacemos/Servicios/VigilanciaSaludPublicaRENAVE/ Enfermedades'Transmisibles/Paginas/-COVID-19.-Informes-previos.aspx

RENAVE, CNE, CNM (ISCIII). (2020). Informe COVID-19 n. ${ }^{\circ} 33$. Recuperado el 29 de mayo 2020 en https://www.isciii.es/QueHacemos/Servicios/VigilanciaSaludPublicaRENAVE/EnfermedadesTransmisibles/Paginas/InformesCOVID-19.aspx

RENAVE, CNE, CNM (ISCIII). (2020). Informe COVID-19 n. ${ }^{\circ}$ 4. Recuperado el 29 de diciembre 2020 en https://www.isciii.es/QueHacemos/Servicios/VigilanciaSaludPublicaRENAVE/EnfermedadesTransmisibles/Documents/INFORMES/Informes\%20COVID-19/Informe\%20COVID-19.\%20N\%c2\%ba\%2054_25\%20de\%20noviembre\%20 de\%202020.pdf

Sociedad Española de Medicina Intensiva, Crítica y Unidades Coronarias (SEMICYUC). (2020). Recomendaciones éticas para la toma de decisiones en la situación excepcional de crisis por pandemia COVID-19 en las unidades de cuidados intensivos. Recuperado el 1 de junio de 2020 en https://semicyuc.org/wp-content/uploads/2020/03/Ética_SEMICYUC-COVID-19.pdf 
Torres-Salinas, D. (2020). Ritmo de crecimiento diario de la producción científica sobre Covid-19. Análisis en bases de datos y repositorios en acceso abierto. El Profesional de la Información, 29, 2, e290215.

United Nations Human Rights Office of the High Commissioner. (2020). COVID-19 and the rights of persons with disabilities guidance. Recuperado el 30 de abril de 2020 en https://www.ohchr.org/Documents/Issues/Disability/COVID-19_and_The_Rights_of_ Persons_with_Disabilities.pdf

Warrillow, S., Austin, D., Cheung, W., Close, E., Holley, A., Horgan, B., Jansen, M., Joynt, G., Lister, P., Moodie, S., Nichol, A., Nicholls, M., Peake, S., Skowronski, G., Streat, S., White, B. y Willmott, L. (2020). ANZICS guiding principles for complex decision making during the COVID-19 pandemic. Critical care and resuscitation. Journal of the Australasian Academy of Critical Care Medicine, 22(2), 98-102. Advance online publication.

World Health Organisation. (2020). Statement on the meeting of the International Health Regulations (2005) Emergency Committee regarding the outbreak of novel coronavirus (2019-nCoV). Recuperado el 19 de junio de 2020 en https://www.who.int/news-room/ detail/23-01-2020-statement-on-the-meeting-of-the-international-health-regulations(2005)-emergency-committee-regarding-the-outbreak-of-novel-coronavirus-(2019)-ncov 